\begin{tabular}{|l|l|c|l}
\hline Eiszeitalter u. Gegenwart & 34 & $29-41$ & Hannover 1984 \\
\end{tabular}

\title{
Schwermineralverteilung und Alter pleistozäner mariner Sedimente in Mittelitalien
}

\author{
UlRich RADTKE \& WOlFGang TILlmanNS*)
}

Heavy mineral distribution, marine sediment, marine sedimentation, beach deposit, section, volcanism, mineral composition.

Southern Maremna, Alto Lacio

Kurzfassung: In den südlichen Maremmen zwischen Civitavecchia und dem Monte Argentario gelang es, mittels der Schwermineralanalyse pleistozäne marine Sedimente unterschiedlichen Alters voneinander abzugrenzen. Anhand der Beschreibung einiger Profile wird gezeigt, daß die ältesten pleistozänen marinen Terrassensedimente durch ein völliges Fehlen vulkanischer Schwerminerale gekennzeichnet sind, d. h. ihre Ablagerung geschah vor 700000-600 000 BP, dem Beginn des vulsinischen Vulkanismus. Sedimente des oberen Mittelpleistozäns und des Jungpleistozäns zeigen ab ca.430000 BP, dem Beginn der Hauptaktivität des Vulkanismus, ein sehr einheitliches Schwermineralspektrum, welches durch die Dominanz des Augits (90-100\%) gekennzeichnet ist. Sedimente, die zwischen Beginn des Vulkanismus und seiner Hauptaktivität abgelagert wurden (700000-600000 bis $430000 \mathrm{BP}$ ), besitzen ein Schwermineralspektrum, welches sich durchschnittlich nur zur Hälfte aus vulkanischen Schwermineralen zusammensetzt.

\section{Heavy Mineral Distribution and Age of Pleistocene Marine Sediments in Middle Italy}

A bstract: In the southern Maremma, Alto Lazio, Middle Italy, heavy mineral studies were used to differentiate several types of beachrock and other marine sediments which are attributed to marine terraces of different age. Horizontal and vertical sections are described and it is pointed out, that the oldest terrace shows an absence of typical volcanic minerals; that means, that their age of development must be older than $600,000-700,000 \mathrm{BP}$, the beginning of the vulsinic volcanism. The Upper Middle and Upper Pleistocene sediments are from the mineralogical point of view very uniform and characterized by $90-100 \%$ augite. Some sediments show a relatively small part of augite (43-70\%); it could be possible, that the sedimentation took place between $600,000-700,000 \mathrm{BP}$ and the main activity of the volcanism in this area at $430,000 \mathrm{BP}$.

\section{Distribuzione dei minerali pesanti e l'età dei sedimenti marini nell'Italia Centrale}

Riassunto: Le ricerche mineralogiche fino qui effettuate sulle panchine di età diversa e sui terrazzi pleistocenici nell'Italia Centrale tra Civitavecchia e Monte Argentario hanno dimostrato che i terrazzi posti superiormente, a causa della mancanza dei tipici minerali vulcanici pesanti devono essere più vecchi di 600.000-700.000 anni: cioè anteriori all'inizio del vulcanismo vulsinico. Un terrazzo mostrava sistematicamente una presenza del $43-70 \%$ di augite, cosicchè sembra possibile che

*) Anschrift der Autoren: Dr. U. RADTKE, Geographisches Institut der Universität Düsseldorf, Universitätsstr. 1, 4000 Düsseldorf 1. - Dr. W. TILlmANNS, Geologisches Institut der Universität zu Köln, Zülpicher Str. 49, 5000 Köln. 
si sia formato tra i $600.000-700.000$ anni ed i 430.000 anni, cioè all'inizio dell'attività principale del vulcanismo in questa regione. I sedimenti pleistocenici medio-superiore mostrano una composizione mineralogica uniforme con concentrazione dominante di augite del 90 fino al $100 \%$.

\section{Einleitung}

Schwermineraluntersuchungen mariner Sedimente in den südlichen Maremmen haben es mit zusätzlicher Hilfe absoluter Altersbestimmungsmethoden ermöglicht, die i.d.R. den vertikalen Abschluß der Terrassensedimente bildenden Beachrockvorkommen, denen jeweils bestimmte Meeresspiegelhochstände zugeordnet werden können, näher zu untergliedern.

Die Schwermineralanalyse setzt in dem Untersuchungsgebiet insofern ,absolute“ Zeitmarken, als den verschiedenen Phasen des vulsinischen Vulkanismus, der mit seiner Tätigkeit zwischen 700000 und 600000 BP begann (PiCHLER 1970; NicOleTti et al. 1979), bestimmte Schwermineralspektren zugeordnet werden können. Proben ohne vulknanische Scherminerale, wie z. B. Augit, Pyroxen oder Hornblende, sind immer altpleistozänen oder pliozänen Alters.

Die Proben der Abschlußsedimente wurden zwei Längsprofilen entnommen, die sich in westöstlicher Richtung von den holozänen bis zu den altpleistozänen Terrassen erstrecken. Zusätzlich wurden an einigen Vertikalprofilen die Sedimente untersucht, die dem jeweiligen Abschlußsediment untergelagert sind.

Für Proben, wo bereits eine absolute Datierung mittels der ${ }^{230} \mathrm{Th} /{ }^{234} \mathrm{U}$ - und der ESR-Altersbestimmungsmethode vorlag, wird diese beigefügt (RADTKE et al. 1981, 1982; RADTKE 1983a, b).

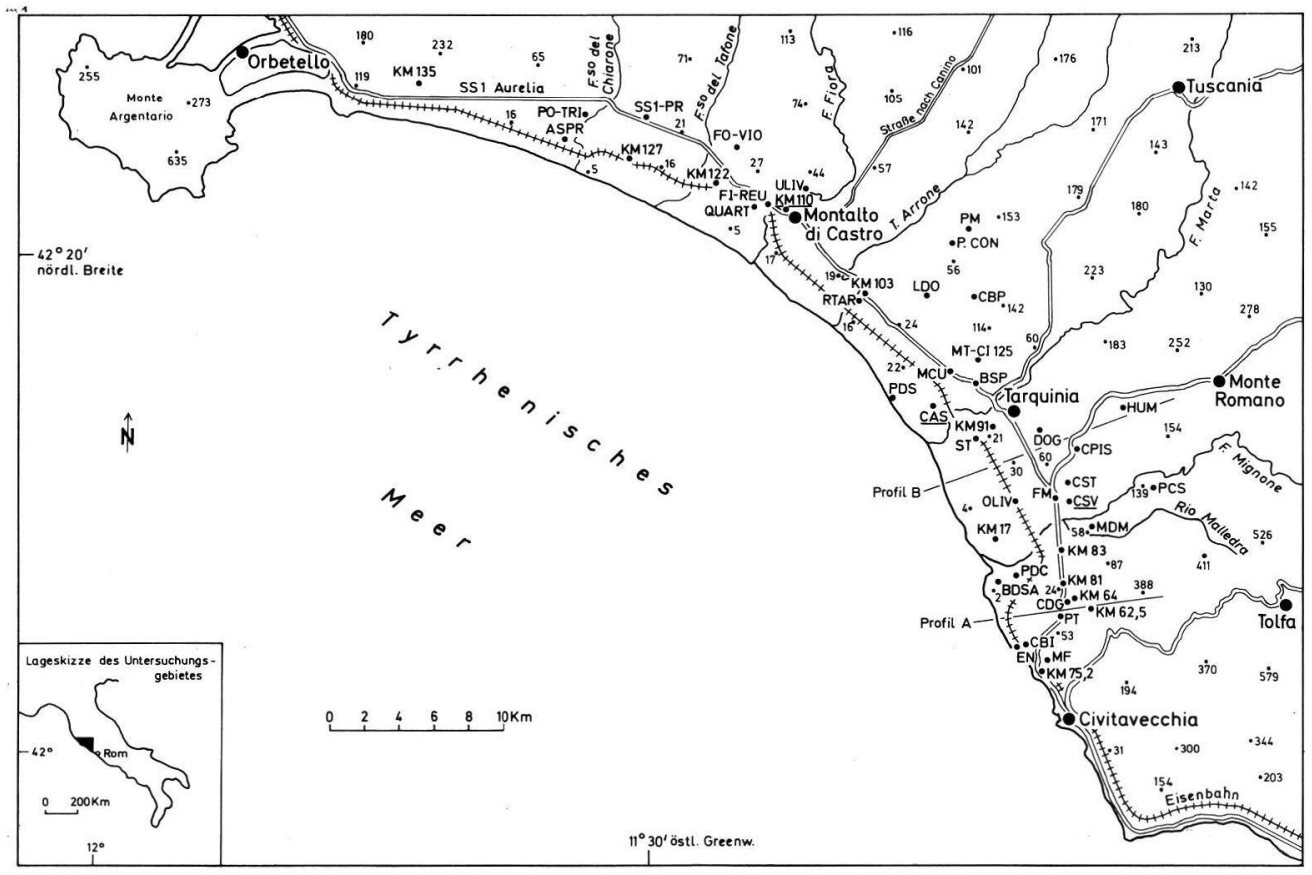

Abb. 1: Lageplan der Probenfundorte 


\section{Schwermineralogische Untersuchungen}

Mittels schwermineralogischer Untersuchungen der in den Längsprofilen A und B sowie den Vertikalprofilen CSV, CAS und KM 110 (s. Abb. 2 bis 5) aufgeschlossenen Sedimente mariner Strandterrassen sollten diese zum einen schwermineralogisch charakterisiert und zum anderen daraufhin untersucht werden, ob sich die Schwermineralführung in Abhängigkeit von Zeit und Lage ändert.

a) Probenvorbereitung

Die Schwermineralführung der Proben wurde nach Kochen der Proben in $\mathrm{HCl}$, Absieben der Fraktion $<0,4 \mathrm{~mm}$ und Abtrennen der Schwerfraktion mittels Bromoform im Scheidetrichter an jeweils 300 transparenten Körnern in den Präparaten bestimmt. Der Anteil opaker Schwerminerale am Gesamtmineralspektrum wurde durch Auszählung von 100 Schwermineralen getrennt bestimmt.

\section{b) Schwermineralführung}

Innerhalb der transparenten Schwerminerale wurden die folgenden Minerale ausgeschieden: Andalusit, Sillimanit, Zirkon, Rutil, Anatas, Turmalin, Augit, Diopsid, braune Hornblende, grüne Hornblende, Titanit, Epidot, Granat, Staurolith und Disthen. Die Unterteilung in metamorphe, stabile, instabile und vulkanische Minerale hat sich für geomorphologisch-geologische Arbeiten bewährt und soll auch hier beibehalten werden, obwohl es selbstverständlich ist, daß z.B. Epidot und Granat, die zu den instabilen Mineralen gezählt werden, natürlich genauso wie der Sillimanit auch metamorphe Minerale darstellen.

In der leichten Fraktion finden sich überwiegend Quarz, Quarzit und silikatische Gesteinsbruchstücke; Feldspäte tauchen nur vereinzelt auf. Signifikante Unterschiede zwischen den einzelnen Proben treten bezüglich des Verhältnisses von Schwermineralen zu Leichtmineralen auf (vgl. Tab. 1 und 2). Eine genauere Analyse der opaken Schwerminerale ist für die Schlußfolgerungen dieser Arbeit irrelevant.

Andalusit und Sillimanit treten nur in zwei Proben auf. Die gegen Transport und insbesondere Verwitterung resistenten Schwerminerale Zirkon, Rutil, Anatas und Turmalin erreichen nur in 13 Proben größere Prozentzahlen (16\% bis maximal 69\%), in den anderen Proben sind sie nur akzessorisch vertreten. In der Gruppe der vulkanischen Schwerminerale (Augit, Diopsid, braune Hornblende und Titanit) dominiert Augit. Diopsid, braune Hornblende und Titanit treten akzessorisch auf. Die Mehrzahl der Proben ist durch die Dominanz von Augit (maximal 100\%) gekennzeichnet. Die instabilen Schwerminerale Epidot, Granat und grüne Hornblende (zusammen maximal 59) erreichen bei Dominanz des Granats in 16 Proben höhere Prozentzahlen. In der Mehrzahl der Proben ist ihr Anteil mit weniger als $10 \%$ genauso unbedeutend wie die vereinzelt auftretenden Staurolithe und Disthene, die hier bei den instabilen Schwermineralen aufgeführt werden.

Hohe Anteile an stabilen (bis 69\%) und instabilen (59\%) Schwermineralen bei fast völligem Fehlen von vulkanischen Schwermineralen zeigen die Proben HUM, PM, CBP (2) und PCS, die den ältesten, höchstgelegenen quartären Strandterrassen entnommen wurden. Das gleichzeitige Auftreten von stabilen und instabilen Schwermineralen schließt eine Verwitterungsauslese der relativ instabilen Schwerminerale aus, auch wenn die Opakenzahl mit bis zu $87 \%$ am Gesamtmineralspektrum sehr hoch ist. Vulkanischer Einfluß zeigt sich über die Führung von Augit (70-43\%), brauner Hornblende sowie Diopsid und Titanit erstmals in den Proben CBP (1), MT-CI 125, CPIS, DOG, P-CON, OLIV, KM 110-2 und ST. Daneben finden sich hohe Anteile an Stabilen (bis 43\%) und untergeordnet Granat (bis 13\%). Auch hier ist eine Verwitterungsauslese vulkanischer Schwerminerale u.a. mangels Verwitte- 
rungserscheinungen an den Mineralen auszuschließen. Die folgenden, tiefer gelegenen Sedimente sind schwermineralogisch durch die Dominanz vulkanischer Schwerminerale (zwischen $84 \%$ und $100 \%$ ) ausgezeichnet.

c) Herkunft der vulkanischen Schwerminerale

Die hier schwermineralogisch untersuchten quartären Strandterrassen liegen jungtertiären Sedimentfolgen auf. An die tertiären Sedimente schließen sich nach E vorwiegend basische Tuffabfolgen quartären Alters und pliozäne Vulkanite unterschiedlichen Chemismus an. Die äußere Umrahmung des Küstentieflandes von Tarquinia bilden im N und S mesozoische Sedimentabfolgen.

Entsprechend der geologischen Situation ist damit zu rechnen, daß die vulkanischen Schwerminerale aus dem Verbreitungsgebiet der Vulkanite (Lago di Bolsena) durch fluviatile und äolische Zufuhr in das Untersuchungsgebiet gelangten. Aus dem Gebiet der tertiären und mesozoischen Schichtfolgen kamen die stabilen, instabilen und metamorphen Schwerminerale vorwiegend durch fluviatilen und marinen Transport.

\section{Schlußfolgerungen}

a) Das Gebiet zwischen Civitavecchia und Fiume Mignone

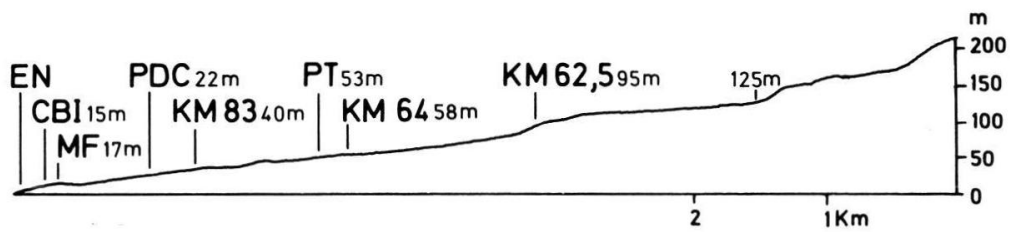

Abb. 2: Terrassenprofil nördlich Civitavecchia (A)

Das Längsprofil beginnt mit zwei direkt übereinanderliegenden Panchinen (ital. Beachrock; Proben EN-U und EN-O), die heute an der Küste der Meeresabrasion ausgesetzt sind; sie lassen sich ebenso wie die landeinwärts folgenden Sedimente, wie z.B. bei CBI, MF, PT und KM 64, schwermineralogisch nicht trennen, denn sie weisen alle einen Augitanteil von 89-98\% auf. EN-O ist auf Grund der absoluten Altersdatierungen wahrscheinlich dem Eutyrrhen II zuzurechnen (Küstenlinie bei ca. 40 m ü.M.), genauso wie CBI, PDC, BDSA, $\mathrm{KM} 81, \mathrm{KM} 83, \mathrm{KM} 75,2$ und MF. EN-U hat sicherlich ein früheres Entstehungsdatum, möglicherweise können die Sedimente, wie bei PT und KM 64, mit dem Eutyrrhen I/PräEutyrrhen (Küstenlinie ca. $75 \mathrm{~m}$ ü.M.) parallelisiert werden.

Die Terrasse ( $95-125 \mathrm{~m})$, die bei KM 62,5 beginnt, setzt sich aus Ablagerungen zusammen, die wesentlich älter sein müssen als die oben beschriebenen, da die vulkanischen Schwerminerale stark zurücktreten. So erscheint ein altpleistozänes, zumindest aber untermittelpleistozänes Alter wahrscheinlich.

Sicherlich altpleistozänes Alter besitzen die Sedimente bei PCS zwischen Rio Melledra und Fiume Mignone (139 m ü.M.), denen vulkanische Minerale gänzlich fehlen.

Leider versagen in diesem Teilgebiet die Möglichkeiten der ,absoluten“ Altersbestimmung weitgehend, da aufgrund der Fossilienarmut der Sedimente bzw. der star- 
ken Kontamination der auffindbaren Proben nur Minimalalter angegeben werden können.

Tab. 1: Schwermineralspektren der Proben aus Profil A

\begin{tabular}{|c|c|c|c|c|c|c|c|c|c|c|c|c|}
\hline Probe & Zirk. & Rut. & Ana. & Turm. & |Vulka & ische & Epid. & Gran. & Opake & $\begin{array}{l}\text { Schwer- } \\
\text { minerale } \\
(\%)\end{array}$ & $\begin{array}{l}\text { Leicht- } \\
\text { minerale } \\
(\%)\end{array}$ & $\begin{array}{c}\text { Altersbestimmungen } \\
\text { (in } \mathrm{ka}=1000 \mathrm{Jahre} \text { ) } \\
\operatorname{ESR}( \pm 25 \%)\end{array}$ \\
\hline PCS & 47 & 5 & 4 & 10 & - & - & 11 & 23 & 75 & - & - & - \\
\hline KM 62,5 & 37 & 8 & 3 & 21 & 11 & - & 8 & 12 & 87 & 0,81 & 99,19 & - \\
\hline KM 64 & 2 & - & - & - & 95 & 1 & - & 2 & 1 & 36,37 & 43,63 & $\geqq 202$ \\
\hline PT & - & - & - & - & 98 & - & - & 2 & 2 & 42,58 & 57,42 & - \\
\hline CDG & - & 1 & - & 3 & 89 & 1 & 1 & 5 & 8 & - & - & - \\
\hline KM 83 & 1 & - & - & - & 93 & 1 & 1 & 4 & 1 & - & - & - \\
\hline KM 81 & - & - & - & 1 & 97 & 2 & - & - & 2 & - & - & $\geqq 105$ \\
\hline PDC & 1 & - & - & - & 94 & 1 & - & 4 & 1 & - & - & $\geqq 121$ \\
\hline BDSA & 1 & - & - & - & 94 & - & - & 5 & 1 & - & - & - \\
\hline MF & 1 & - & - & - & 94 & - & - & 5 & 2 & 38,74 & 61,26 & $\geqq 115$ \\
\hline KM 75,2 & 2 & - & 1 & - & 90 & - & - & 1 & 2 & - & - & - \\
\hline CBI-C & - & - & - & 1 & 97 & 1 & - & 1 & 1 & 55,30 & 44,70 & $\geqq 83$ \\
\hline EN-O & 2 & - & - & - & 92 & 1 & - & 5 & 3 & 58,81 & 41,19 & - \\
\hline EN-U & 3 & - & - & - & 93 & - & - & 4 & 3 & 46,42 & 53,58 & - \\
\hline
\end{tabular}

Zirk. $=$ Zirkon

Rut. = Rutil

Ana. = Anatas

Turm. $=$ Turmalin

$$
\begin{aligned}
& \text { Aug. }=\text { Augit } \\
& \text { Tit. }=\text { Titanit } \\
& \text { Epid. }=\text { Epidot } \\
& \text { Gran. }=\text { Granat }
\end{aligned}
$$

b) Das Gebiet zwischen Fiume Mignone und Torrente Arrone

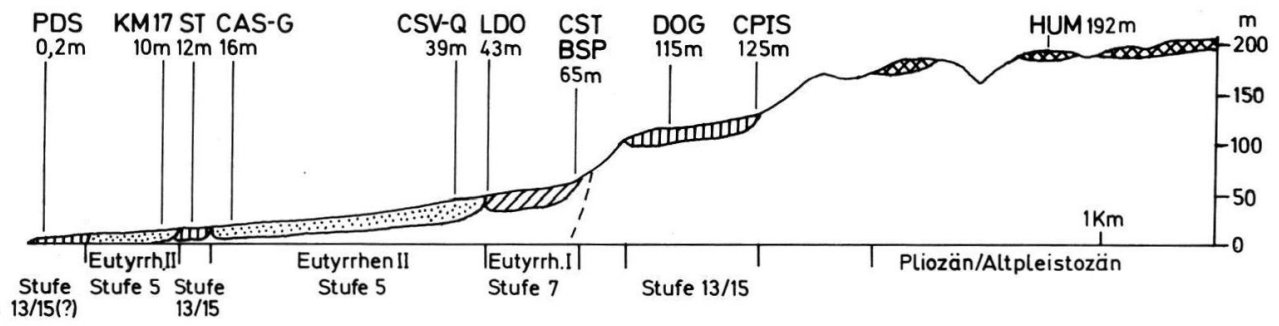

Abb. 3: Terrassenprofil bei Tarquinia (B)

Das Ergebnis der Schwermineralanalyse zeigt wiederum, daß die jungpleistozänen Sedimente nicht näher zu untergliedern sind: 


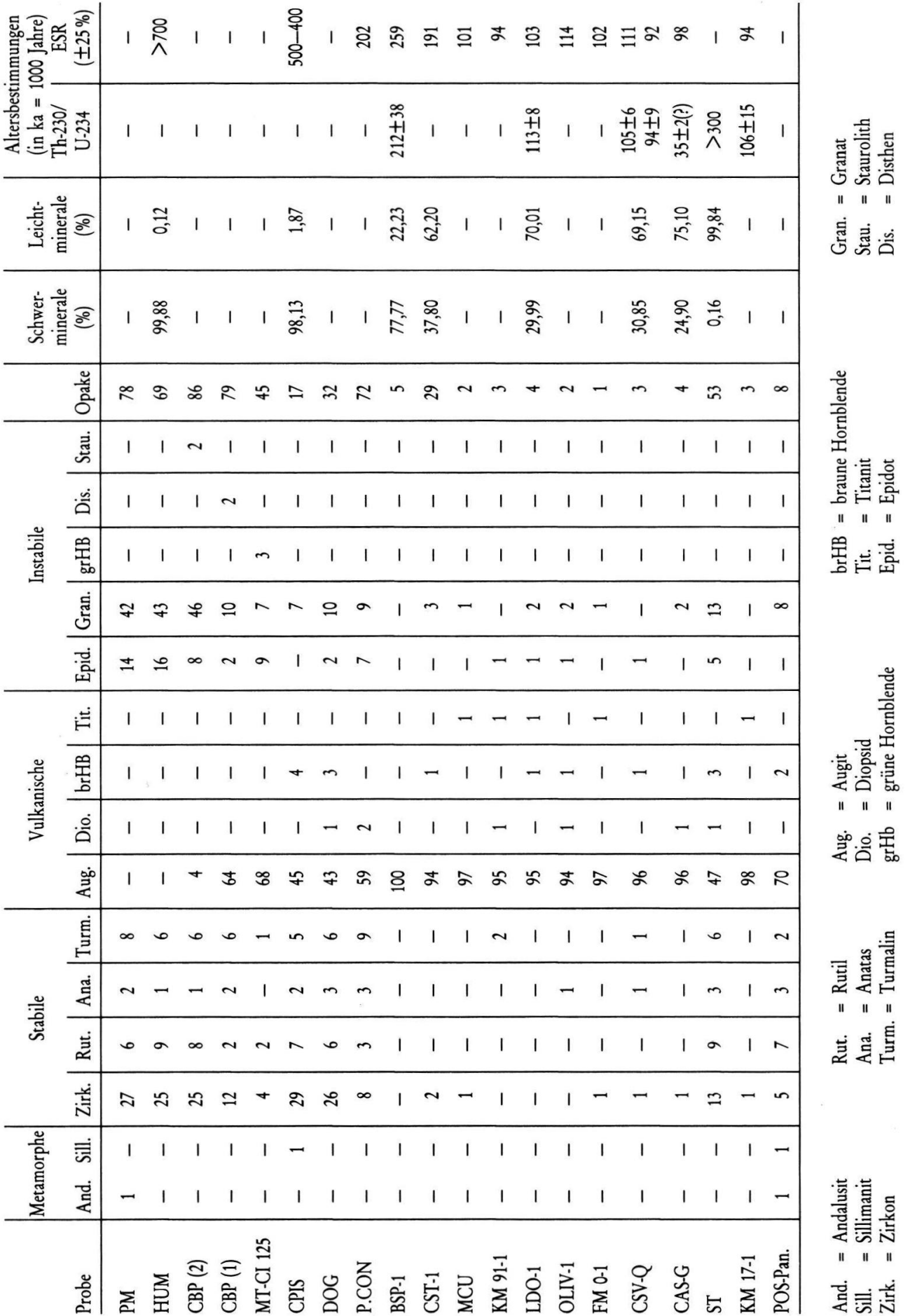

Tab. 2: Schwermineralspektren der Proben aus dem Gebiet zwischen F. Mignone und T. Arrone 
So findet man im Längsprofil B die Relikte des Eutyrrhen II-Meeres (120000 bis 100000 BP) bis ca. 40-45 m ü.M. (Proben KM 17, CAS-G, CSV-Q, LDO etc.) und die des Eutyrrhen I-Meeres (220000—200000 BP) bis ca. $65 \mathrm{~m}$ ü.M. (Proben CST, BSP, CSV-A) mit einer fast identischen mineralogischen Zusammensetzung.

Die große Einheitlichkeit im Schwermineralspektrum innerhalb des EutyrrhenKomplexes verdeutlicht auch folgendes Vertikalprofil bei C.S. Vincenzo (CSV, entspricht den Punkten $G$ und $H$ in RadTKe et al. 1982).

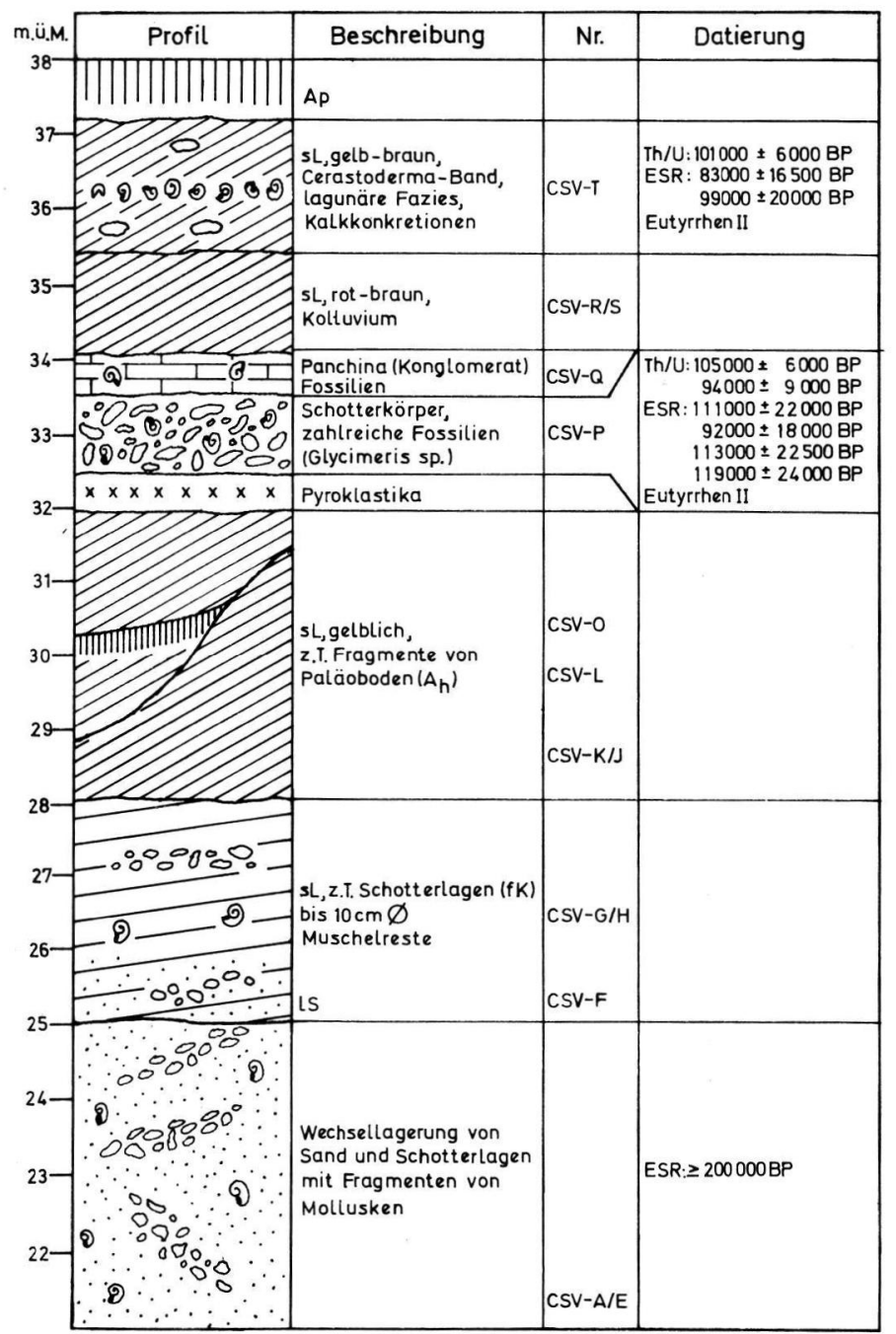

Abb. 4: Profil bei Casale S. Vincenzo (CSV) 
Die einzelnen Schichten zeigen nachstehende Verteilung der Schwerminerale:

Tab. 3: Schwermineralverteilung im Profil CSV

\begin{tabular}{|c|c|c|c|c|c|c|c|c|c|c|}
\hline \multirow[b]{2}{*}{ Probe } & \multirow[b]{2}{*}{ Zirkon } & \multirow{2}{*}{$\begin{array}{l}\text { Stabile } \\
\text { Rutil }\end{array}$} & \multirow{2}{*}{$\begin{array}{l}\text { Tur- } \\
\text { malin }\end{array}$} & \multicolumn{4}{|c|}{$\begin{array}{l}\text { Vulkanische } \\
\text { br. Horn- }\end{array}$} & \multicolumn{2}{|c|}{ Instabile } & \multirow[b]{2}{*}{ Opake } \\
\hline & & & & Augit & Diposid & blende & Titanit & Epidot & Granat & \\
\hline CSV-T & - & - & 1 & 97 & - & - & - & 1 & 1 & 3 \\
\hline CSV-S & - & 1 & - & 96 & 1 & 1 & - & - & 1 & 2 \\
\hline CSV-Q & 1 & - & 1 & 96 & - & 1 & - & 1 & - & 3 \\
\hline CSV-P & - & - & 1 & 97 & 1 & - & - & 1 & - & 1 \\
\hline CSV-O & - & - & - & 99 & 1 & - & - & - & - & 1 \\
\hline CSV-N & - & - & - & 94 & 1 & 1 & - & 1 & 3 & - \\
\hline CSV-L & - & - & - & 97 & - & - & - & 1 & 2 & 5 \\
\hline CSV-K & - & - & - & 98 & 1 & - & - & - & 1 & 2 \\
\hline CSV-J & 1 & - & - & 95 & 1 & 1 & - & 1 & 1 & 6 \\
\hline CSV-H & 1 & 1 & - & 97 & - & - & - & - & 1 & 9 \\
\hline CSV-F & 5 & - & 1 & 79 & - & 1 & - & 3 & 11 & 10 \\
\hline CSV-D & 3 & 1 & - & 89 & - & 1 & 1 & - & 5 & 9 \\
\hline CSV-E & 1 & 1 & 1 & 95 & - & - & - & 1 & 1 & 5 \\
\hline CSV-G & - & - & $\cdot 2$ & 94 & 1 & - & 1 & - & 2 & 7 \\
\hline CSV-A & 2 & - & - & 92 & 1 & 1 & - & 1 & 3 & 13 \\
\hline
\end{tabular}

Sicherlich finden lokal sich verändernde Strömungsverhältnisse des Meeres ihren Niederschlag im Schwermineralspektrum, doch scheint dieser Einfluß sowie die Aufarbeitung von altpleistozänen/pliozänen Sedimenten so gering gewesen zu sein, daß es als gesicherte Erkenntnis gelten darf, daß die Sedimente des Tyrrhen durch eine eindeutige Dominanz des Augits gekennzeichnet sind; in Kap. 4 wird gezeigt, daß gleiches auch für die Ablagerungen des oberen Mittelpleistozäns gilt.

Wie deutlich sich die Diskordanz zu unterlagernden pliozänen Schichten im Schwermineralspektrum widerspiegel, soll das folgende Vertikalprofil (CAS, entspricht Punkt C in RADTKE el al. 1982) mitsamt Tabelle verdeutlichen:

Tab. 4: Schwermineralverteilung im Profil CAS

\begin{tabular}{|c|c|c|c|c|c|c|c|c|c|}
\hline \multirow[b]{2}{*}{ Probe } & \multicolumn{4}{|c|}{ Stabile } & \multicolumn{2}{|c|}{ Vulkanische } & \multicolumn{2}{|c|}{ Instabile } & \multirow[b]{2}{*}{ Opake } \\
\hline & Zirkon & Rutil & Anatas & Turmalin & Augit & Dioposid & Epidot & Granat & \\
\hline CAS-H & - & - & - & 1 & 95 & - & 1 & 3 & 1 \\
\hline CAS-G & 1 & - & - & - & 96 & 1 & - & 2 & 4 \\
\hline CAS-E & - & - & - & - & 97 & - & - & 3 & 2 \\
\hline CAS-D & 16 & 7 & - & 4 & 21 & - & 11 & 41 & 40 \\
\hline CAS-B & 21 & 10 & 2 & 7 & - & - & 9 & 51 & 72 \\
\hline CAS-A & 24 & 13 & 1 & 9 & - & - & 14 & 39 & 65 \\
\hline
\end{tabular}

Probe CAS-D gehört eindeutig zu dem Pliozänkomplex. Der Augitanteil von $21 \%$ ist durch eine partielle Einarbeitung von jungem Material bei der Transgression des Eutyrrhenmeeres zu erklären.

Aus dem Rahmen fallen scheinbar die Spektren der Proben PDS (0,2 m ü.M.) und ST (12 m ü.M., Probe B in RadTKE et al. 1982). Von der Höhenlage ableitend, würde man sie 


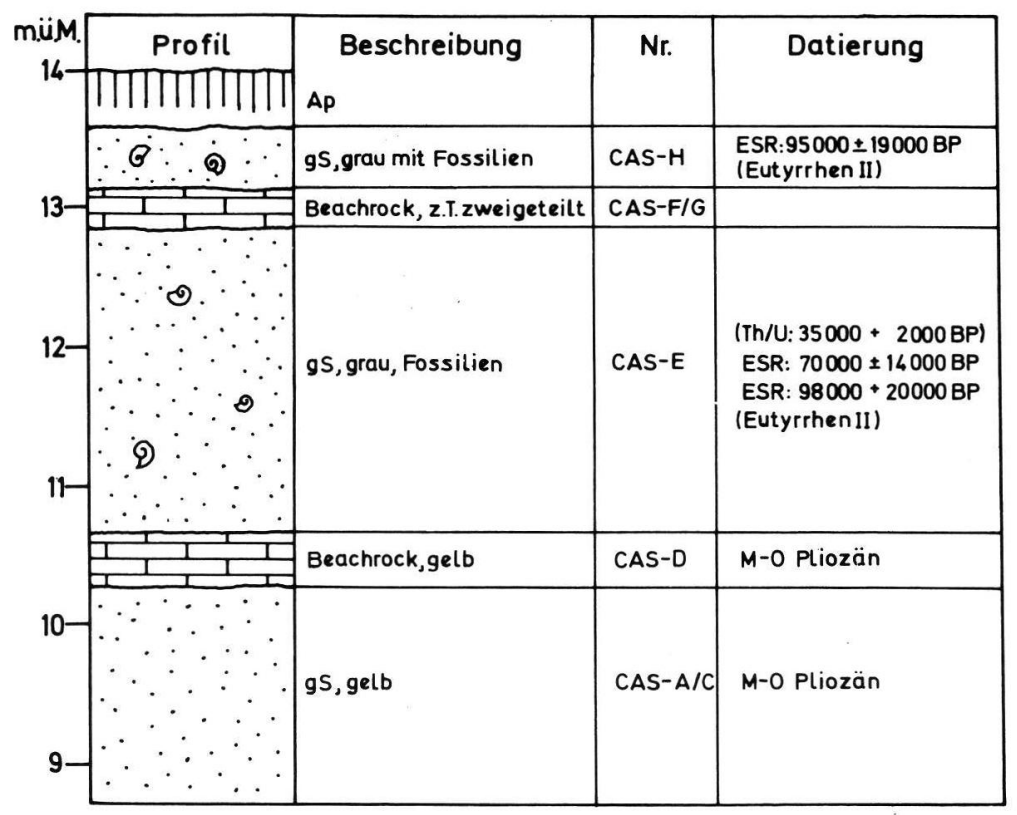

Abb. 5: Profil bei il Casalino (CAS)

sicherlich dem Eutyrrhen II zuordnen. Vergleicht man aber die Spektren mit denen von DOG, CPIS, MT-CI 125 und P.CON in ca. 100-125 m ü.M., so lassen sich verblüffende Übereinstimmungen feststellen: der Augitanteil ist nicht mehr absolut dominant, sondern schwankt zwischen 43 und $70 \%$. Das ${ }^{230} \mathrm{Th} /{ }^{234} \mathrm{U}$-Alter (älter als $300000 \mathrm{BP}$ ) von Probe ST belegt zusätzlich, daß es sich um einen präeutyrrhenen Meeresspiegelhochstand handelt, dessen fossile Küstenlinie sich heute bei ca. $125 \mathrm{~m}$ ü.M. auf dem Horst von Tarquinia befindet (DAi PRA 1978; RADTKe 1983 a, b). Die Sedimente aus der Zeit zwischen dem Beginn des vulsinischen Vulkanismus und seiner ersten Hauptaktivität (NICOLETTI et al. 1979) sind somit durch eine deutlich verringerte Dominanz des Augits gekennzeichnet. Eindeutig unterscheiden sie sich aber von den jüngeren Sedimenten durch einen signifikant höheren Anteil an den opaken, stabilen und instabilen Schwermineralen. Die stratigraphischen Verhältnisse und die Altersdatierungen schließen aus, daß es sich um rein lokale Erscheinungen der verstärkten Aufarbeitung pliozänen Materials handelt.

Das oberste Glied der Terrassentreppe (Proben HUM, PM, CBP (2)) wird von ConATO \& DAI PRA (1981) in das Altpleistozän gestellt, die schwermineralogische Untersuchung der Proben aus den Terrassenabschlußsedimenten dieses Transgressionszyklus' (Serie della Macchia della Turchina, FAZZINI et al. 1972) bestätigt zumindest, daß die Ablagerung vor $700000-600000$ BP, d.h. im Altpleistozän/Pliozän stattfand. Eine genauere Einordnung ist zur Zeit noch nicht möglich.

\section{c) Das Gebiet zwischen Torrente Arrone und Monte Argentario}

Am Unterlauf des T. Arrone kündigt sich der Wechsel in den tektonischen Verhältnissen an. Bei RTAR verläuft die Küstenlinie des Eutyrrhen II nunmehr in ca. $25 \mathrm{~m}$ ü.M., nördlich dieses Punktes konnte sie nicht mehr zweifelsfrei nachgewiesen werden. Die Hebungstendenz, die im Raum von Tarquinia eindeutig dominierte, schwächt sich in Richtung 
Monte Argentario immer mehr ab. Bei KM 135 der Via Aurelia konnten pleistozäne Sedimente nur noch bis zu einer Höhe von 15-20 m ü.M. (Lago di Burano-Düne) ausgegliedert werden; i.d.R. erreichen die marinen Ablagerungen nur 5-10 m ü.M. Nördlich von Montalto di Castro verlaufen die verschiedenen fossilen Küstenlinien des Alt-, Mittel- und Jungpleistozäns in einem nicht immer genau zu differenzierenden Saum im Bereich von ca. 25-40 m ü.M. Wir finden hier also keine ,horizontale" West-Ost-Abfolge der verschieden alten marinen Terrassensedimente, sondern ein rein ,,vertikales“ Übereinander eines Terrassenstapels (AMBROSETTI et al. 1981, RADTKE et al. 1981). Dies gilt gleichermaßen für die Aufschlüsse KM 122 und KM 127 an der Eisenbahnstrecke Rom-Genua und KM 103 und KM 110 der Via Aurelia. Letztgenanntes Vertikalprofil soll im folgenden näher erläutert werden:

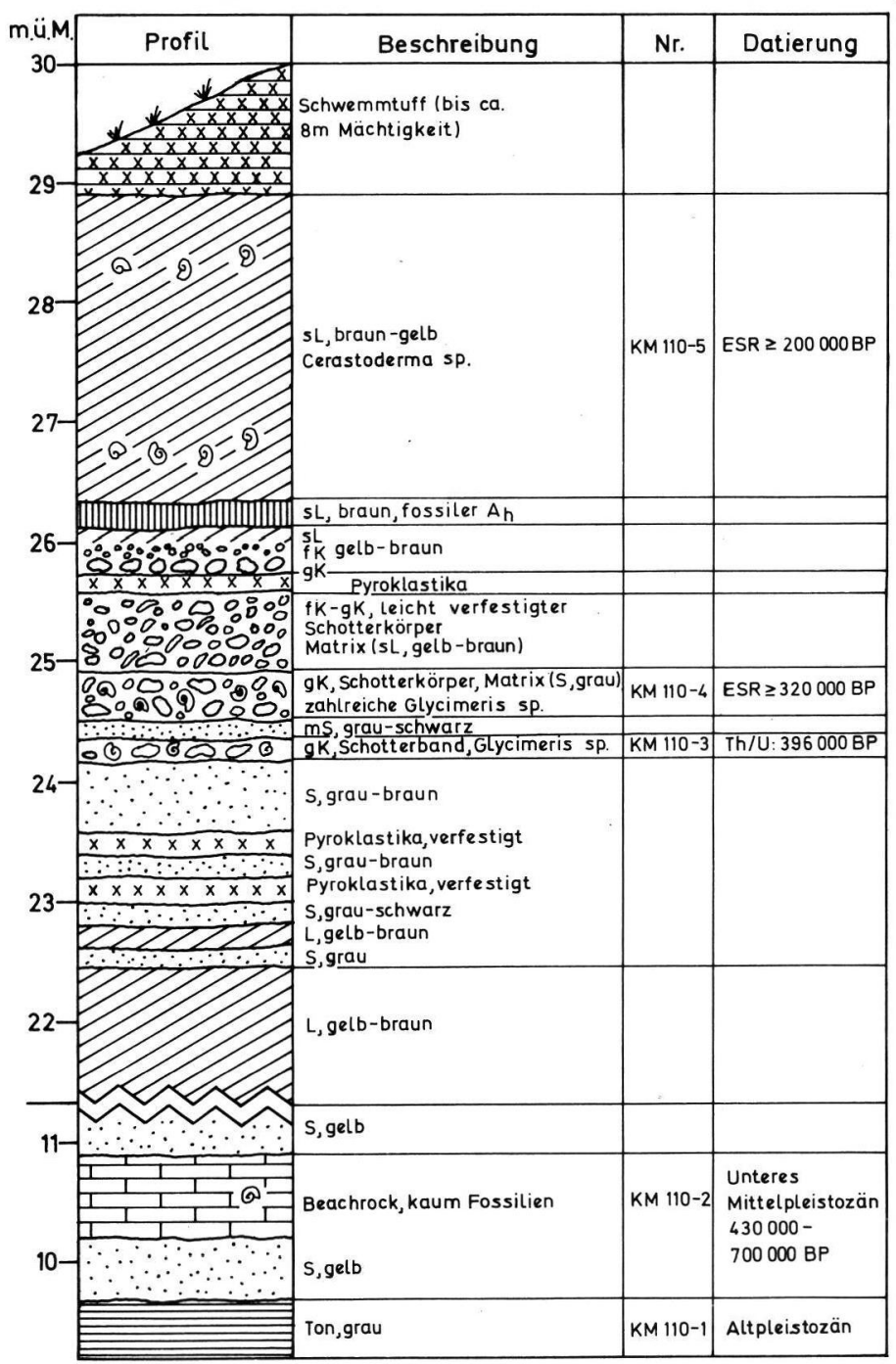

Abb. 6: Profil bei KM 110 der SS1 Aurelia 
Tab. 5: Schwermineralverteilung im Profil KM 110

\begin{tabular}{|c|c|c|c|c|c|c|c|c|c|c|c|c|}
\hline \multirow[b]{2}{*}{ Probe } & \multicolumn{4}{|c|}{ Stabile } & \multicolumn{3}{|c|}{ Vulkanische } & \multicolumn{4}{|c|}{ Instabile } & \multirow[b]{2}{*}{ Opake } \\
\hline & Zirk. & Rut. & Ana. & Turm. & Aug. & brHB & Tit. & Epid. & Gran. & Dis. & Stau. & \\
\hline KM 110-5 & - & - & - & 1 & 94 & 1 & 1 & - & 3 & - & - & 5 \\
\hline KM 110-4 & 1 & - & - & 1 & 96 & - & 1 & - & 1 & - & - & 3 \\
\hline KM 110-3 & 1 & - & - & - & 97 & - & - & - & 2 & - & - & 1 \\
\hline KM 110-2 & 6 & 3 & 1 & 6 & 67 & 3 & - & 1 & 10 & 1 & 2 & 61 \\
\hline
\end{tabular}

Wie im vorhergehenden Kapitel schon bemerkt, ist es bestätigt, daß die Dominanz des Augits im Schwermineralspektrum auch für Sedimente des oberen Mittelpleistozäns gilt (vgl. auch Profil MDM in RaDTKe 1983a, 89f.).

In der folgenden Tabelle sind einige repräsentative Analysen des nördlichen Teilgebietes zusammengestellt, die die obigen Aussagen untermauern. Unsichere bzw. „fehlerhafte“ Alter, die an Cerastoderma sp. gemessen wurden (RADTKE et al. 1981; RADTKE 1983a, b), werden in dieser Tabelle nicht berücksichtigt. Es handelt sich ausschließlich um Werte, die auf einer Datierung von Glycimeris sp. beruhen.

Tab. 6: Schwermineralspektren der Proben aus dem Gebiet zwischen T. Arrone und M. Argentario

\begin{tabular}{|c|c|c|c|c|c|c|c|c|c|c|c|c|c|c|c|c|}
\hline \multirow[b]{2}{*}{ Probe } & \multicolumn{4}{|c|}{ Stabile } & \multicolumn{4}{|c|}{ Vulkanische } & \multicolumn{4}{|c|}{ Instabile } & \multirow[b]{2}{*}{$\begin{array}{l}\text { Alte- } \\
\text { rit }\end{array}$} & \multirow[b]{2}{*}{ Opake } & \multicolumn{2}{|c|}{$\begin{array}{c}\text { Alters- } \\
\text { bestimmungen } \\
\text { (in ka=1000 Jahre) }\end{array}$} \\
\hline & Zirk. & Rut. & Ana. & Turm. & Aug. & Dio. & brHB & Tit. & Epid. & Gran. & Dis. & Stau. & & & $\begin{array}{l}\text { Th-230/ } \\
\text { U-2234 }\end{array}$ & $\begin{array}{c}\text { ESR } \\
( \pm 25 \%\end{array}$ \\
\hline ULIV & 9 & 2 & 3 & 8 & 60 & - & 2 & 1 & 2 & 12 & 1 & - & - & 75 & - & - \\
\hline KM 110-2 & 6 & 3 & 1 & 6 & 67 & - & 3 & - & 1 & 10 & 1 & 2 & - & 61 & - & - \\
\hline ASPR & 2 & - & - & 1 & 93 & - & - & - & 1 & 3 & - & - & - & 19 & - & - \\
\hline KM 135 & 1 & - & - & - & 96 & - & - & 2 & - & 1 & - & - & - & 3 & - & - \\
\hline PO-TRI & 1 & - & - & - & 97 & - & 1 & - & - & 1 & - & - & - & 5 & - & - \\
\hline SS1-PR & - & - & 1 & - & 98 & - & 1 & - & - & - & - & - & - & 2 & - & - \\
\hline KM 127(3)-A & 1 & 1 & - & - & 91 & 1 & 2 & - & - & 4 & - & - & - & 6 & - & 305 \\
\hline KM $122(3)-A$ & 3 & - & - & - & 84 & - & - & 2 & 1 & 7 & - & - & 3 & $\begin{array}{c}38 \\
\text { (Magne } \\
\text { tit) }\end{array}$ & $158 \pm 12$ & 163 \\
\hline FO-VIO & 1 & - & - & 1 & 98 & - & - & - & - & - & - & - & - & 1 & - & - \\
\hline QUART-A & - & 1 & - & - & 95 & - & 1 & 2 & - & 2 & - & - & - & 4 & - & 235 \\
\hline FI-REU & - & - & - & - & 96 & - & - & 1 & 1 & 2 & - & - & - & 5 & - & - \\
\hline KM 103-D & - & - & 1 & - & 97 & - & - & - & - & 2 & - & - & - & 2 & $\geqq 151$ & $\geqq 200$ \\
\hline RTAR-In & 1 & - & - & - & 98 & - & - & 1 & - & - & - & - & - & 1 & $115 \pm_{12}^{15}$ & 102,3 \\
\hline
\end{tabular}

\section{Schlußbemerkungen}

Es kann festgehalten werden, daß es aufgrund der Interpretation der Schwermineralanalysen möglich ist, im Küstentiefland von Tarquinia zwischen den Monti della Tolfa und dem Monte Argentario marine (und auch terrestrische) Sedimente anhand ihres Gehaltes an vulkanischen Schwermineralen drei größeren geologischen Epochen zuzuordnen: 
1. (Pliozän)/Altpleistozän

Der Zeitraum vor ca. 700000-600000 BP ist gekennzeichnet durch die Absenz vulkanischer Schwerminerale, wie Augit, Diopsid, Titanit, brauner und grüner Hornblende. Durch das direkte Nebeneinander von stabilen und instabilen Schwermineralen ist eine selektive Verwitterung der vulkanischen Minerale auszuschließen.

2. Unteres Mittelpleistozän

Zwischen 700000-600000 und ca. 430000 BP, d.h. zwischen dem Beginn und der ersten Hauptaktivität des vulsinischen Vulkanismus erreichen die vulkanischen Schwerminerale einen Anteil im Schwermineralspektrum, der bei durchschnittlich 50-70\% liegt, es kommt nie zu einer eindeutigen Dominanz.

3. Oberes Mittelpleistozän und Jungpleistozän

In der Zeit nach der Hauptaktivität des Vulkanismus erreichen die vulkanischen Minerale die absolute Dominanz: Vornehmlich der Augit erreicht 90-100\% im Schwermineralspektrum.

Durch junge tektonische Bewegungen sind die quartären Ablagerungen in den verschiedenen Bereichen des Untersuchungsgebietes in unterschiedliche Höhenlagen gebracht worden. Es ist also nicht möglich, eine fossile Küstenlinien in einer konstanten Höhenlage im Küstentiefland zu verfolgen. Frühere Arbeiten auf rein altimetrischer Grundlage mußten somit zu Fehlinterpretationen führen, als man versuchte, bestimmte Terrassenniveaus in das klassische System des marinen Quartärs im mediterranen Raum einzuordnen. Nunmehr erscheint es möglich, mittels der Schwermineralanalyse in diesem Küstengebiet Mittelitaliens, deren Aussagen im wesentlichen durch die ,absolute“ Altersbestimmung bestätigt wurden, relativ schnell und sicher zu einer ersten Grobgliederung der quartären Sedimente zu kommen. Dieser ersten Analyse können dann im Einzelfall natürlich detaillierte Untersuchungen folgen.

\section{Schriftenverzeichnis}

Ambrosetti, P., Bartolini, C. \& Bosi, C. (1981): L'evoluzione geologica e morfologica quaternaria dell'area adiacente la bassa valle del Fiume Fiora (Viterbo). - Geogr. Fis. Dinam. Quat., 4: 104-124, Turin.

Alberti, A., Bertini, M., Del Bono, G.L., Nappi, G. \& Salvati, L. (1971): Note illustrative della Carta Geologica d'Italia F. 142 (Civitavecchia). 139 S., Neapel.

Bonadonna, F.P. (1967): Studi sul Pleistocene del Lazio. III. Linee di costa lungo il litorale di Tarquinia (Lazio settentrionale). - Geol. Rom., 6: 121-135, 8 Abb., 1 Tab.; Rom.

Conato, V. \& Dai Pra, G. (1981): Livelli marini Pleistocenici e Neotettonica fra Civitavecchia e Tarquinia (Italia Centrale). - Geol. Rom., 19: 181-194, 2 Abb., 3 Tab.; Rom.

DAI PrA, G. (1978): Linea di costa tirrenianae del litorale laziale dal Fiume Tevere a Tarquinia. Geol. Appl. Idrog., 13: 1-10, 1 Abb.; Bari.

Fazzini, P., Gelmini, R., Mantovani, M.P. \& Pellegrini, M. (1972): Geologia dei Monti della Tol$\mathrm{fa}$ (Lazio settentrionale, Province de Viterbo e Roma). - Mem. Soc. Geol. Ital., 11 (1): 65-144; Pisa.

IKeYA, M. \& OhmuRA, K. (1981): Dating fossil shells with Electron Spin Resonance. - Jour. Geol., 89: 247-251, 3 Abb.; Chicago.

Nicoletti, M., Petrucciani, C., Piro, M. \& Trigila, R. (1979): Nuove datazioni vulsinie per uno schema di evoluzione della attività vulcanica: il quadrante nord - occidentale. - Period. Mineral., 48: 153-165; Rom. 
Pichler, H. (1970): Italienische Vulkangebiete I. - Sammlung Geologischer Führer, 51: 258 S., 48 Abb., 9 Tab., 9 Taf., 5 Beil.; Berlin-Stuttgart.

Radtke, U., Hennig, G.J., Linke, W. \& MÜngersdorf, J. (1981): ${ }^{230} \mathrm{Th} /{ }^{234} \mathrm{U}$ - und ESR-dating problems of fossil shells in Pleistocene marine terraces (Northern Latium, Central Italy). - Quaternaria, 23: 37-50, 4 Abb., 3 Tab.; Rom.

- $\quad$, Hennig, G.J. \& Mangini, A. (1982): Untersuchung zur Chronostratigraphie mariner Terrassen in Mittelitalien $-{ }^{230} \mathrm{Th} /{ }^{234} \mathrm{U}$ - und ESR-Datierungen an fossilen Muscheln. - Eiszeitalter und Gegenwart, 32: 49-56, 1 Abb., 2 Tab.; Öhringen/ Württ.

- (1983a): Genese und Altersstellung der marinen Terrassen zwischen Civitavecchia und Monte Argentario (Mittelitalien) unter besonderer Berücksichtigung der Elektronenspin-ResonanzAltersbestimmungsmethode. - Düsseldorfer Geographische Schriften, 22: 180 S., 50 Abb., 11 Tab., 29 Photos, 2 Beil.,; Düsseldorf.

- (1983b): Elektronenspin-Resonanz-Altersdatierungen fossiler Mollusken in ,Beachrock'Generationen Latiums, Mittelitalien. - Essener Geographische Arbeiten, 6: 201-215, 8 Abb., 1 Tab.; Paderborn. 
